PROCEEDINGS OF THE

AMERICAN MATHEMATICAL SOCIETY

Volume 125, Number 9, September 1997, Pages 2803-2808

S 0002-9939(97)03935-X

\title{
BRANCHED COVERS ALONG REAL PARTS
}

\author{
SHUGUANG WANG
}

(Communicated by Ronald A. Fintushel)

\begin{abstract}
It is shown that the double cover of a Kähler surface branched along the fixed point set of an anti-holomorphic involution admits a symplectic structure.
\end{abstract}

In [4] Donaldson suggests studying the quotient manifold of a Kähler surface under an anti-holomorphic involution. It turns out, in case the involution is not free, that such a quotient does not have a symplectic structure, let alone a Kähler structure, by using Kronheimer-Mrowka [10] or Morgan-Szabo-Taubes [12] and Taubes [14]. (More precisely, the fixed point set of the involution is assumed to be orientable of genus bigger than 1 . Then its image on the quotient manifold violates the "adjunction" inequality of [10], [12]; thus the quotient has vanishing SeibergWitten invariants and by [14] does not have symplectic structures.) Going in a different direction, Akbulut [1] and Finashin [7] have shown directly that, in many cases, the quotient can be decomposed into a connected sum of $\mathbb{C P}^{2}, S^{2} \times S^{2}$ and their reverses. In case the anti-holomorphic involution is free, the situation becomes slightly different in that the quotient is no longer decomposable by Wang [18] and is even irreducible from Kotschick [9], although the quotient still does not admit any symplectic structure [18].

In contrast with the above, we consider here another construction of smooth 4-manifolds arising from anti-holomorphic involutions and we will show that these manifolds have symplectic structures.

Consider in general a co-dimension 2 submanifold $\Sigma$ of a smooth $n$-manifold $X$ ( $\Sigma$ may be non-orientable or disconnected). If the homology class $[\Sigma]=0 \in$ $H_{n-2}\left(X, \mathbb{Z}_{2}\right)$, then it is a familiar fact that there exists a double cover $\widetilde{X} \rightarrow X$ branched precisely along $\Sigma$, which will be unique up to equivalence if in addition $H_{1}\left(X, \mathbb{Z}_{2}\right)=0$. Recall also that a smooth map $\sigma: X \rightarrow Y$ between two almost complex manifolds is called anti-holomorphic if $\sigma_{*} \circ J_{X}=-J_{Y} \circ \sigma_{*}$ for the almost complex structures $J_{X}, J_{Y}$. When $X=Y$ and $\sigma^{2}=i d$, the fixed point set of $\sigma$ is called a real part. The main result in the note is the following:

Theorem. Let $X$ be a Kähler surface with $H_{1}(X, \mathbb{Z})=\{1\}$ and $\Sigma \subset X$ be the real part of an anti-holomorphic involution $\sigma$ on $X$. Suppose that $\Sigma$ consists of orientable surfaces only and $[\Sigma]=0 \in H_{2}\left(X, \mathbb{Z}_{2}\right)$. Then the double cover $p: \widetilde{X} \rightarrow X$ branched along $\Sigma$ admits a symplectic structure.

Received by the editors March 22, 1996 and, in revised form, April 30, 1996.

1991 Mathematics Subject Classification. Primary 57R55, 57R57, 57N13; Secondary 14P25.

Key words and phrases. Branched cover, real part, symplectic structure.

(C) 1997 American Mathematical Society 
Proof. The idea is to perturb the Kähler form on $X$ to a symplectic form so that $\Sigma$ becomes a symplectic submanifold; this is basically due to Gompf [7, Lemma 1.6]. Then pull back the symplectic form from $X$ to $\widetilde{X}$ and perturb it again to get symplectic form on $\widetilde{X}$; the construction here is adapted from Thurston [15] (compare with McDuff $[11, \S 3]$ ).

By using $\tilde{X}$, there is a canonical choice of a semi-orientation on the connected components $\left\{\Sigma_{i}\right\}_{1}^{m}$ of $\Sigma$, namely a pair of opposite orientations on $\Sigma$. According to Viro $[17,2.4$.A] the semi-orientation can be characterized as the only one such that $[ \pm \Sigma] \in H_{2}(X, \mathbb{Z})$ are equal to $\pm 2 a$ where $a$ is some element in the image of the inverse Hopf homomorphism $p^{!}: H_{2}(X / \sigma, \mathbb{Z}) \rightarrow H_{2}(X, \mathbb{Z})$. This implies in particular $\left[\Sigma_{i}\right] \neq 0$ in $H_{2}(X, \mathbb{Z})$ for all $i$. It follows that there is a linear functional $f: H_{2}(X, \mathbb{R}) \rightarrow \mathbb{R}$ satisfying $f\left(\left[\Sigma_{i}\right]\right) \neq 0$ for all $i$ (induction on $m$ ). With one of the two orientations in the semi-orientation on $\Sigma$ which will be fixed from now on, we have all $f\left(\left[\Sigma_{i}\right]\right)>0$. By taking the dual of $f$, there is a closed 2-form $\xi \in H_{D R}^{2}(X)$ with $\int_{\Sigma_{i}} \xi>0$. So one can choose a symplectic form $\omega_{i}$ on each $\Sigma_{i}$ such that $\int_{\Sigma_{i}} \omega_{i}=\int_{\Sigma_{i}} \xi$. Thus there is a 1-form $\gamma_{i}$ on $\Sigma_{i}$ satisfying $\omega_{i}=\xi+d \gamma_{i}$ on $\Sigma_{i}$. Since $\amalg \Sigma_{i} \subset X$ is a closed subset, one can extend $\left\{\gamma_{i}\right\}$ to a 1 -form $\gamma$ on $X$.

Let $\omega$ be the Kähler form on $X$. One can assume $\sigma^{*} \omega=-\omega$ (otherwise consider the Kähler form $\sigma^{*} \omega-\omega$, which is compatible with the same almost complex structure as $\omega$ ). It follows that $\omega$ restricts to a zero form on $\Sigma$. (Namely $\Sigma$ is a Lagrangian submanifold in $X$, a standard fact in real algebraic geometry.)

Fix a $t>0$ and consider a perturbation $\omega^{\prime}=\omega+t(\xi+d \gamma)$, which is obviously a closed form on $X$. For small $t, \omega^{\prime}$ is non-degenerate, as $\omega$ is non-degenerate and non-degeneracy is an open condition. Thus $\omega^{\prime}$ is a symplectic form on $X$, whose restriction to each $\Sigma_{i}$,

$$
\left.\omega^{\prime}\right|_{\Sigma_{i}}=\left.\omega\right|_{\Sigma_{i}}+\left.t(\xi+d \gamma)\right|_{\Sigma_{i}}=t\left(\left.\xi\right|_{\Sigma_{i}}+d \gamma_{i}\right)=t \omega_{i}
$$

is non-degenerate. In other words, we obtain a symplectic form $\omega^{\prime}$ on $X$ as a perturbation of $\omega$ under which $\Sigma$ is a symplectic submanifold.

Let $\widetilde{\Sigma}=p^{-1}(\Sigma)$. We proceed to construct a closed 2-form $\eta$ on $\widetilde{X}$ such that it is non-degenerate on fibers of the normal bundle $\left.W \subset T \widetilde{X}\right|_{\tilde{\Sigma}}$ of $\widetilde{\Sigma}$ in $\widetilde{X}$, and such that the fibers $W_{x}, T_{x} \widetilde{\Sigma}$ are $\eta$-orthogonal for each $x \in \widetilde{\Sigma}$. Consider the pull-back $\widetilde{\xi}=p^{*} \xi$ of the closed 2-form $\xi$ constructed in an early paragraph. This is a closed 2-form on $\widetilde{X}$ with $\int_{\widetilde{\Sigma}} \widetilde{\xi}=\int_{\Sigma} \xi>0$ (meaning $\int_{\widetilde{\Sigma_{i}}} \widetilde{\xi}>0$ for all $i$; the subscript $i$ will continue to be suppressed to save space). So there is again a Kähler (symplectic) structure on $\widetilde{\Sigma}$ such that its Kähler form $\Omega$ satisfies $\Omega=\widetilde{\xi}+d \widetilde{\gamma}$ on $\widetilde{\Sigma}$ for some 1 -form $\widetilde{\gamma}$. With $\Omega$ on the base $\widetilde{\Sigma}$, choose any holomorphic structure on the bundle $W \rightarrow \widetilde{\Sigma}$. Thus the total space of $W$ becomes a Kähler manifold whose Kähler form will also be denoted by $\Omega$. Note that the fibers $W_{x}, T_{x} \widetilde{\Sigma}$ are $\Omega$-orthogonal by definition of $\Omega$ (that is, $\Omega(u, v)=0$ for any $u \in W_{x}, v \in T_{x} \widetilde{\Sigma}$ ). Extend $\widetilde{\xi}, \widetilde{\gamma}$ to $W$ by pull-back.

Let $N$ be a tubular neighborhood of $\widetilde{\Sigma}$. By embedding $N$ into $W, N$ inherits a closed 2-form $\widehat{\Omega}$ and a 1-form $\widehat{\gamma}$ from $\Omega, \widetilde{\gamma}$, such that $\widehat{\Omega}=\widetilde{\xi}+d \widetilde{\gamma}$ on $\widetilde{\Sigma}$. The important properties of $\widehat{\Omega}$ are that for any $x \in \widetilde{\Sigma}$, the fibers $W_{x}, T_{x} \widetilde{\Sigma}$ are $\widehat{\Omega}$-orthogonal, and $\widehat{\Omega}$ is non-degenerate on $W_{x}$, since the fibers are holomorphic submanifolds of the total space of $W$. Extend $\widehat{\gamma}$ to a 1 -form $\delta$ on $\widetilde{X}$. Then $\eta=\widetilde{\xi}+d \delta$ is the desired closed 2-form on $\tilde{X}$. 
Now consider $\omega_{t}=p^{*} \omega^{\prime}+t \eta$, which is a closed 2-form on $\widetilde{X}$, where $t>0$. Pick up any $x \in \widetilde{\Sigma}$; we show that $\omega_{t}$ is non-degenerate on $T_{x} \widetilde{X}$ for small $t$. Indeed, as $p^{*} \omega^{\prime}$ is non-degenerate on $T_{x} \widetilde{\Sigma}$ (recall $\Sigma$ is $\omega^{\prime}$-symplectic), $\omega_{t}$ is also for small $t$. For the same $t$, it is straightforward to check that $\omega_{t}$ is non-degenerate on $T_{x} \tilde{X}$, in view of the properties of $\eta$ and that $p^{*} \omega^{\prime}(u, v)=0$, if either $u$ or $v$ belongs to $W_{x}$. Thus $\omega_{t}$ will be non-degenerate in a neighborhood of $x$ in $\tilde{X}$. By compactness of $\widetilde{\Sigma}, \omega_{t}$ is non-degenerate in a neighborhood $R \subset N$ of $\widetilde{\Sigma}$ for all small $t>0$.

On the other hand, given any neighborhood $S \subset R$ of $\widetilde{\Sigma}, \omega_{t}$ is non-degenerate on $\widetilde{X} \backslash S$ for all small $t>0$, as $p^{*} \omega^{\prime}$ is non-degenerate there. Thus once $t$ is small enough, $\omega_{t}$ is non-degenerate on $\widetilde{X}=R \cup(\widetilde{X} \backslash S)$ hence a symplectic form on $\widetilde{X}$.

Remark. As initial evidence for the Theorem, one can verify directly that the cover $\widetilde{X}$ admits an almost complex structure. Indeed, let $\widetilde{K}=p^{*} K_{X}$ be the pull-back of the canonical line bundle $K_{X}$ of $X$ and $\hat{K}=\widetilde{K} \otimes P D[\widetilde{\Sigma}]$. Then from the usual Euler characteristic and signature formulas for double covers, one has that

$$
\begin{aligned}
& \hat{K}^{2}-\left(2 \chi_{\widetilde{X}}+3 \tau_{\widetilde{X}}\right) \\
& =2\left[K_{X}^{2}-\left(2 \chi_{X}+3 \tau_{X}\right)\right]+2\left(\chi_{\Sigma}+\Sigma^{2}+K_{X} \cdot P D[\Sigma]\right) .
\end{aligned}
$$

But $K_{X}^{2}-\left(2 \chi_{X}+3 \tau_{X}\right)=0, \chi_{\Sigma}+\Sigma^{2}=0$ (since $J_{X}$ induces an orientation reversing isomorphism between the tangent and normal bundles of $\Sigma$ ) and $\left.K_{X} \cdot P D[\Sigma]\right)=0$ (since $\left.\sigma^{*} K_{X}=-K_{X}\right)$. Thus $\hat{K}^{2}-\left(2 \chi_{\widetilde{X}}+3 \tau_{\widetilde{X}}\right)=0$. Since also $c_{1}(\hat{K}) \equiv w_{2}(\widetilde{X})$ modulo 2 (from $w_{2}(\widetilde{X})=p^{*} w_{2}(X)+P D[\widetilde{\Sigma}]$ ), an old result of Hopf and Hirzebruch says that $\hat{K}$ is the "canonical" bundle of some almost complex structure on $\widetilde{X}$.

To give some examples satisfying the conditions in the Theorem, recall that any real part $\Sigma \subset X$ satisfies the generalized Harnack inequality

$$
\sum_{q} \operatorname{dim} H_{q}\left(\Sigma, \mathbb{Z}_{2}\right) \leq \sum_{q} \operatorname{dim} H_{q}\left(X, \mathbb{Z}_{2}\right) ;
$$

$\Sigma$ is called an M-real part if the equality holds, see e.g. Wilson [19].

Corollary. If $X$ is a spin Kähler surface with $H_{1}(X, \mathbb{Z})=\{1\}$, and $\sigma$ is an antiholomorphic involution whose real part $\Sigma$ is an $M$-real surface, then the branched cover $\widetilde{X} \rightarrow X$ exists and has a symplectic structure.

Proof. Since $H_{1}(X, \mathbb{Z})=\{1\}$, there is only one spin structure on $X$, which must be preserved by $\sigma$. This implies that $\Sigma$ is orientable by Edmonds [5].

As $\Sigma$ is an M-surface, the induced map $\sigma_{*}: H_{2}\left(X, \mathbb{Z}_{2}\right) \rightarrow H_{2}\left(X, \mathbb{Z}_{2}\right)$ is the identity, [19]. On the other hand $[\Sigma] \in H_{2}\left(X, \mathbb{Z}_{2}\right)$ is characteristic with respect to $\sigma_{*}$, namely for any $y \in H_{2}\left(X, \mathbb{Z}_{2}\right)$, the intersection numbers obey $[\Sigma] \cdot \sigma_{*} y=y \cdot y$, see Arnold [2]. Combining with $\sigma_{*}=i d$ and $y \cdot y=0$ (since $X$ is spin), we have $[\Sigma] \cdot y=0$ for all $y \in H_{2}\left(X, \mathbb{Z}_{2}\right)$, which implies $[\Sigma]=0$ in $H_{2}\left(X, \mathbb{Z}_{2}\right)$.

So the conditions in the Theorem are satisfied and the Corollary follows.

Constructing M-real parts is an important problem in real algebraic geometry. For instance, it is a classical theorem of Harnack that for every degree $d$, there is a projective curve $C \subset \mathbb{C P}^{2}$ whose real part is an M-real algebraic curve. Take $d$ even and consider the double cover $X$ of $\mathbb{C P}^{2}$ branched along $C$. Then the complex conjugation on $\mathbb{C P}^{2}$ can be lifted to an anti-holomorphic involution on $X$ which has an M-real part. Another source of anti-holomorphic involutions on Kähler 
surfaces with M-real parts is given in Viro [16], which constructs, for every degree, a projective surface $X \subset \mathbb{C P}^{3}$ with M-real surface. In both cases, except for a few lower degrees, the real parts have at least one component which is not a sphere.

From the proof, it is possible to extend the Theorem to a finite-fold cover $\widetilde{X} \rightarrow X$ branched along $\Sigma=$ Fix $\sigma$, where $\sigma: X \rightarrow X$ is an involution on a symplectic manifold $(X, \omega)$, which is anti-holomorphic with respect to some $\omega$-compatible almost complex structure. (The last condition is equivalent to saying that $\sigma$ is antisymplectic, namely $\sigma^{*} \omega^{\prime}=-\omega^{\prime}$, where the symplectic form $\omega^{\prime}=\sigma^{*} \omega-\omega$ is the average of $\omega$.) However practically all known examples are covered by the Theorem, especially by the Corollary.

It appears to be an interesting question whether the double cover $\widetilde{X}$ in the Theorem admits a Kähler structure. We hope to consider elsewhere this question as well as other general branched covers, in connection with Seiberg-Witten invariants. Here we are content with observing the following Proposition. It implies that if $\Sigma$ has a non-sphere component, then $\widetilde{X}$ is not diffeomorphic to any Kähler surface that is a holomorphic double cover with branched locus diffeomorphic to $\Sigma$.

Proposition. Suppose $X$ is a minimal surface of general type and $\Sigma \subset X$ is the real part of some anti-holomorphic involution $\sigma$. Then there is no complex surface $Y$ together with a diffeomorphism $f: X \rightarrow Y$ such that $f(\Sigma) \subset Y$ is a complex submanifold, unless the following happen: All components of $\Sigma$ are spheres and $Y$ has a (-2)-curve.

Proof. As before let $K_{X}$ be the canonical bundle of $X$. Then $\sigma^{*} K_{X}=-K_{X}$ and hence $K_{X} \cdot P D\left[\Sigma_{i}\right]=0$ for all components $\Sigma_{i}$ of $\Sigma$.

Suppose that there is a diffeomorphism $f: X \rightarrow Y$ to a complex surface $Y$ with $f(\Sigma) \subset Y$ a complex curve. By Friedman-Morgan [8], one of the $\pm\left(f^{-1}\right)^{*} K_{X}$ is the canonical bundle of $Y$ and $Y$ is also minimal of general type. Since

$$
\begin{aligned}
& \left(f^{-1}\right)^{*} K_{X} \cdot P D\left[f\left(\Sigma_{i}\right)\right] \\
& =\left(f^{-1}\right)^{*} K_{X} \cdot\left(f^{-1}\right)^{*}\left(P D\left[\Sigma_{i}\right]\right) \\
& =K_{X} \cdot P D\left[\Sigma_{i}\right]=0,
\end{aligned}
$$

by Barth-Peters-Van de Ven [3, page 208, Corollary (2.3)], $f\left(\Sigma_{i}\right)$ must be a (-2)curve. This means that $\Sigma_{i}$ is a sphere and $Y$ contains a $(-2)$-curve $f\left(\Sigma_{i}\right)$.

Note that in the Proposition above, the condition that $\Sigma$ be a real part may be weakened to one of the following purely topological conditions:

$$
K_{X} \cdot P D\left[\Sigma_{i}\right]=0 \text { or } \chi_{\Sigma_{i}}+\Sigma_{i}^{2}=0 .
$$

(Under the second condition, use the adjunction formula on $Y$ to deduce that $f\left(\Sigma_{i}\right)$ are $(-2)$-curves. Since $\chi_{\Sigma_{i}}+\Sigma_{i}^{2}=0$ is also preserved by any homeomorphism, the diffeomorphism $f$ in the Proposition under this condition may be replaced by a homeomorphism provided $Y$ is assumed to be of general type.) For another remark about the Proposition, by a theorem of S.-T. Yau, saying that $Y$ has (-2)-curves is equivalent to saying that $Y$ has no Kähler-Einstein metrics, which excludes for instance hypersurfaces of degree $>3$ in $\mathbb{C P}^{3}$.

As a future application, it will be of independent interest to use the Proposition above to construct "exotic" 2-dimensional knots inside smooth 4-manifolds. Another consequence is the following: 
Corollary. In the Proposition, assume in addition that not all components of $\Sigma$ are spheres. Then $\sigma$ is not smoothly equivalent to any holomorphic involution on another complex manifold.

Proof. The case when $\Sigma$ is empty follows from [18]. If $\Sigma \neq \emptyset$, supposing the existence of the equivalence, then $\Sigma$ is mapped onto the fixed point set of the holomorphic involution, which is then a complex submanifold. By the Proposition above, all components of $\Sigma$ are spheres.

Ruan [13] has shown, by using different methods ( $G$-invariant Seiberg-Witten invariants), that more generally the Corollary remains true even if $\Sigma$ consists of spheres. In fact the author learned from Yongbin Ruan another direct proof of this which was recently pointed out by Peter Kronheimer: Since $\sigma^{*} K_{X}=-K_{X}$, for any diffeomorphism $f: X \rightarrow Y$ we have

$$
\left(f \circ \sigma \circ f^{-1}\right)^{*}\left[\left(f^{-1}\right)^{*} K_{X}\right]=-\left(f^{-1}\right)^{*} K_{X} .
$$

As $\left(f^{-1}\right)^{*} K_{X}= \pm K_{Y}$ is preserved by every holomorphic involution on $Y$, we see that $f \circ \sigma \circ f^{-1}$ cannot be a holomorphic involution on $Y$.

\section{ACKNOWLEDGEMENT}

The author would like to thank Qi Zhang for some helpful discussions.

\section{NOTE ADDED IN PROOF}

Prompted by an example of G. Mikhalkin, the author realized that in the proof of the Theorem, Viro's characterization of semi-orientations of $\Sigma$ does not imply $\left[\Sigma_{i}\right] \neq 0 \in H_{2}(X, \mathbb{Z})$ when $\Sigma$ is connected. Thus in the statement of the Theorem, we need to insert the following words before the last sentence: When $\Sigma$ is connected, we assume also $[\Sigma] \neq 0$.

\section{REFERENCES}

1. S. Akbulut, On quotients of complex surfaces under complex conjugation, J. Reine Angew. Math. 447 (1994), 83-90. MR 95c:57030

2. V. Arnold, On the location of ovals of real algebraic plane curves, involutions on 4dimensional smooth manifolds, and the arithmetic of integral quadratic forms, Funct. Analy. Appl. 5 (1971), 1-9. MR 44:3999

3. W. Barth, C. Peters and A. Van de Ven, Compact complex surfaces, Springer-Verlag Berlin Heidelberg, 1984. MR 86c:32026

4. S. Donaldson, Yang-Mills invariants of four-manifolds, In Geometry of low-dimensional manifolds. Vol 1, pp 5-41. S.K. Donaldson and C.B. Thomas Eds., Cambridge University Press, 1990. MR 93f:57040

5. A. Edmonds, Orientability of fixed point sets, Proc. Amer. Math. Soc. 82 (1981), 120-125. MR 82h:57032

6. R. Gompf, A new construction of symplectic manifolds, Ann. Math. 142 (1995), 527-595. MR 96g:57025

7. S. Finashin, Decomposability of quotients by complex conjugation for complete intersection surfaces, Revista Mat. Univ. Comp. Madrid (to appear).

8. R. Friedman and J. Morgan, Algebraic surfaces and Seiberg-Witten invariants, Preprint (1995).

9. D. Kotschick, On irreducible four-manifolds, Preprint (1995).

10. P. Kronheimer and T. Mrowka, The genus of embedded surfaces in the projective plane, Math. Res. Letters 1 (1994), 797-808. MR 96a:57073

11. D. McDuff, Examples of simply-connected symplectic non-Kählerian manifolds, J. Diff. Geom. 20 (1984), 267-277. MR 86c:57036 
12. J. Morgan, Z. Szabo and C. Taubes, A product formula for the Seiberg-Witten invariants and the generalized Thom conjecture, Preprint (1995).

13. Y. Ruan, Virtual neighborhoods and monopole equations, Preprint (1996).

14. C. Taubes, The Seiberg-Witten invariants and symplectic forms, Math. Research Letters 1 (1994), 809-822. MR 95j:57039

15. W. Thurston, Some simple examples of symplectic manifolds, Proc. Amer. Math. Soc. 55 (1976), 467-469. MR 53:6578

16. O. Viro, Construction of M-surfaces, Funct. Analy. Appl. 13 (1979), 212-213. MR 80j:14034

17. Complex orientations of real algebraic surfaces, Topology of manifolds and varieties, Ed. O. Viro. Advances in Soviet Mathematics, Vol 18. Published by American Mathematical Society. (1994), 261-284. MR 96f:14068

18. S. Wang, A vanishing theorem for Seiberg-Witten invariants, Math. Res. Letters 2 (1995), 305-310.

19. G. Wilson, On Hilbert sixteenth problem, Topology 17 (1978), 53-73. MR 58:16684

Department of Mathematics, University of Missouri, Columbia, Missouri 65211

E-mail address: sw@wang.math.missouri.edu 